\title{
T-DNA activation-tagged mycelia producing fruiting bodies-specific triterpenoids in Antrodia cinnamomea - a medicinal fungus
}

\author{
Chen ECF ${ }^{1}$, Agrawal $\mathrm{DC}^{1} *$, Wang $\mathrm{HY}^{1}$, Chen $\mathrm{WP}^{1}$ and Tsay $\mathrm{HS}^{1,2} *$ \\ ${ }^{1}$ Department of Applied Chemistry, Chaoyang University of Technology, Taichung, Taiwan \\ ${ }^{2}$ Department of Agronomy, National Chung Hsing University, Taichung, Taiwan
}

Chen ECF, Agrawal DC, Wang HY, Chen WP, Tsay HS. 2016 - T-DNA activation-tagged mycelia producing fruiting bodies-specific triterpenoids in Antrodia cinnamomea - a medicinal fungus. Mycosphere 7(5), 602-611, Doi 10.5943/mycosphere/7/5/6

\begin{abstract}
Antrodia cinnamomea, a medicinal fungus native to Taiwan is used as a traditional Chinese medicine for the treatment of several critical illnesses including cancer. It was reported that the medicinal properties of this fungus are mainly due to the presence of certain triterpenoids in fruiting bodies, which are generally absent in mycelia. Fruiting bodies of A. cinnamomea carry an exorbitantly high price due to a short supply and ever increasing demand. In the present study, we report the development of activation tagged transgenic mycelia lines of $A$. cinnamomea. HPLC analysis of three transgenic lines showed production of both mycelia and fruiting bodies (FB) specific triterpenoids (dehydrosulphurenic acid, dehydroeburicoic acid, antcin A, B and $\mathrm{C}$ ). The discovery of FB-specific triterpenoids in these transgenic lines of A. cinnamomea has significance since mycelia of this fungus are becoming increasingly popular as a functional food for the treatment of several diseases including cancer.
\end{abstract}

Key words - A. cinnamomea - antcin A - antcin B - antcin C - dehydroeburicoic acid dehydrosulphurenic acid - transgenic fungus

\section{Introduction}

Antrodia cinnamomea belonging to Basidiomycetes group of fungi is native to Taiwan. In Chinese, it is known as 'Niuchangchih.' A. cinnamomea grows as a parasite on the inner heartwood wall of rotting empty trunk of camphor tree (Cinnamomum kanehiraih Hay) (Lauraceae), which is the only natural endemic host tree for this medicinally important fungus. Brownish orange, perennial fruiting bodies of $A$. cinnamomea have a strong camphor aroma and have been used by Taiwanese aboriginal tribes for centuries for the treatment of food, alcohol, and drug intoxication, diarrhea, abdominal pain, hypertension, itching, and to enhance liver function (Tsai \& Liaw 1982). Scientific research on the taxonomic identification and binomial nomenclature of A. cinnamomea and potential applications of this fungus as medicine began in the last three decades. Fruiting bodies of A. cinnamomea have been reported to contain bioactive compounds having cytotoxic (Nakamura et al. 2004), anti-inflammatory (Shen et al. 2004), and neuroprotective (Chen et al. 2006) activities and generally are recognized as a precious traditional medicine and functional food material. Owning to a short supply due to slow growth, excessive collection in wild, difficult to culture in vitro and ever increasing demand fruiting bodies of A. cinnamomea carry an exorbitantly high price, about US\$20,000 per Kg (Chiang \& Chiang 2013). 
In earlier reports, mycelia of $A$. cinnamomea could be cultivated in vitro, but these did not produce certain fruiting bodies (FB) specific triterpenoids, i.e. zhankuic acids A,B,C, or antcin K. These compounds could be isolated only from fruiting bodies of A. cinnamomea, and are considered to be effective compounds for curing certain illnesses (Shen et al. 2004). However, mycelia of $A$. cinnamomea are now recognized as a health promoting product. During the last decade, a wide range of biological functions of the mycelium of A. cinnamomea have been recognized such as anti-oxidation (Huang \& Mau 2007, Mau et al. 2004, Song \& Yen 2002), antiinflammatory (Chen et al. 2007, Liu et al. 2007, Shen et al. 2004), antitumor (Hseu et al. 2007, Hsu et al. 2005, 2007, Kuo et al. 2006, Liu et al. 2004, Song et al. 2005, Yang et al. 2006), hepatoprotective (Hsiao et al. 2003, Song \& Yen 2003), vasorelaxative (Wang et al. 2003) and anti-hepatitis $\mathrm{B}$ virus (Lee et al. 2002). More recently, Tsai et al. (2015) demonstrated that methanolic extracts of wood-cultured fruiting bodies and solid-state-cultured mycelia of A. camphorata have excellent anti-inflammatory activities and thus have great potential as an addition to hydrocolloid dressings. In another report, the anti-metastatic effects of antrodan, the glycoprotein from A. cinnamomea mycelia, through direct actions and indirect immunomodulatory effects in Lewis lung carcinoma (LLC) have been demonstrated (Fa et al. 2015). A total of 78 compounds, including terpenoids, benzenoids, lignans and benzoquinone derivatives have been identified in the mycelia of $A$. cinnamomea. Among terpenoids group, the triterpenoids are considered as the most biologically active components (Geethangili \& Tzeng 2011). Therefore, induction of bioactive compounds in the mycelia of $A$. cinnamomea was considered most needed. Activation tagging, a method to generate dominant mutations by random insertion of enhancer sequences into the genome can produce gain-of-function or loss-of-function mutants. Due to its efficiency and a high percentage of single T-DNA insertion, T-DNA activation tagging using cauliflower mosaic virus (CaMV) $35 \mathrm{~S}$ enhancers is the preferred method. This activation tagging approach has been used successfully in several plant species, such as Arabidopsis (Kardailsky et al. 1999), Catharanthus roseus (Van der Fits et al. 2001), tomato (Mathews et al. 2003), poplar (Busov et al. 2003), and rice (Hsing et al. 2007). In the case of fungi, ours was the first report on the development of activation tagged mycelia of $A$. cinnamomea (Chen et al. 2009).

In the present study, we report HPLC analysis of three transgenic mycelia lines of $A$. cinnamomea to discover possible fruiting bodies specific triterpenoids (dehydrosulphurenic acid, dehydroeburicoic acid, antcin A, B and C) in them.

\section{Materials \& Methods}

\section{Fungal strain}

Wild type (WT) Antrodia cinnamomea strain BCRC 36401 (Fig.1 A) was procured from the Bioresources Collection and Research Center (BCRC), Food Industry Research and Development Institute, Hsinchu, Taiwan. Three activation tagged transgenic lines $\mathrm{H}, \mathrm{J} 1$, and $\mathrm{J} 2$ of $A$. cinnamomea, hereinafter referred as GMM (genetically modified mycelia) (Fig.1. B-D), developed in our laboratory were selected for the present investigation on the basis of higher mycelia growth rates compared to other GMM lines. AD-1, a 35S antisense mutant of DMATS (Dimethylallyl tryptophan synthase) was created (Fig.1. F) and used as a control.

\section{Culture medium and conditions}

The wild type strain BCRC 36401 was maintained on a medium consisting of $2 \%$ glucose, $2 \%$ malt extract, $0.1 \%$ peptone and $2 \%$ agar hereinafter referred as malt-extract-agar (MEA) medium (Chang \& Chou 2004). GMM lines and AD-1 were cultured on MEA medium supplemented with hygromycin $(40 \mathrm{mg} / \mathrm{L})$ as a selection pressure. Mycelia cultures were maintained in petridishes $\left(90 \times 15 \mathrm{~mm}\right.$ ), each containing $25 \mathrm{ml}$ of medium and incubated at $25{ }^{\circ} \mathrm{C}$ in dark for 4 weeks. These cultures were used as a source of inoculum for further study. Before carrying out further investigation, all GMM lines along with control (wild type mycelia, WT) were subjected to polymerase chain reaction (PCR) and gel electrophoresis analysis to confirm insertion 
of hphII (950 bp) and gus (650 bp) genes in all GMM lines. AD-1 was also subjected to PCR and gel electrophoresis analysis to confirm its DMATS gene knock-out status. D-f: 5'CCAGGCTTGGACGTGTCGACG -3'; D-r: 5'-GGGCGTACTCGCGTTTATGGGG-3' were used as primers, and the PCR was performed under the following conditions: $94{ }^{\circ} \mathrm{C}$ for $5 \mathrm{~min}$ followed by 35 cycles at $94{ }^{\circ} \mathrm{C}$ for $30 \mathrm{sec}, 61{ }^{\circ} \mathrm{C}$ for $30 \mathrm{sec}$ and $72{ }^{\circ} \mathrm{C}$ for $1 \mathrm{~min}$., and ACTIN was used as an internal control (Actin-f: 5'-ACGTTCAACGCGCCTGCCTTC-3', and Actin-r: 5'GGTGGTGTGGAACTGGTACCCACG-3'). The optimum transformation conditions for activation tagged GMM lines was $5 \times 10^{8} \mathrm{cfu} \mathrm{\textrm {m } ^ { - }}{ }^{-}$Agrobacterium cells (Agrobacterium tumefaciens strain EHA105, carrying a binary vector pTAG8), $1 \mathrm{mM}$ acetosyringone, 25-days-old mycelia at $0.2 \mathrm{~g}$ $\mathrm{ml}^{-1}$, and a co-culture period of 6 days. A detailed procedure of development of activation tagged GMM lines of this fungus has been given in our previous report (Chen et al. 2009).

\section{HPLC Analysis - reagents and conditions}

Fruiting bodies (FB) of wild type and all GMM lines were subjected to HPLC analysis. Total five triterpenoid compounds, two lanostane-type (dehydrosulphurenic acid and dehydroeburicoic acid) and three ergostane-type triterpenoids (antcin A, B and C) were estimated. HPLC grade methanol and acetonitrile were purchased from J.T. Baker (Phillipsburg, NJ, USA). Pump (L-2130), autoinjector (L-2200) and diode array detector system (L-2450) used were from Hitachi, Japan. Column: Symmetry Water Column C18 (5 $\mu \mathrm{m}, 4.6 \times 250 \mathrm{~mm})$, Phenomenex Luna $5 \mathrm{u}$ silica ( 5 microns, $250 \times 4.60 \mathrm{~mm}$ and pure water (Millipore, Milford, MA, USA) were used for the analysis carried out at a room temperature. The mobile phase for lanostane-type triterpenoids, a gradient eluting with $0.01 \%$ phosphoric acid $\left(\mathrm{H}_{3} \mathrm{PO}_{4}\right) / 100 \%$ acetonitrile $\left(\mathrm{CH}_{3} \mathrm{CN}\right)$ at different time intervals (min) as follows: (40:60:0; 35:65:20; 10:90:60; 10:90:65; 40:60:70; 40:60:80). The sample injection volume was $20 \mu \mathrm{l}$ at a flow rate of $1 \mathrm{ml} / \mathrm{min}$ and a detection wavelength of $244 \mathrm{~nm}$. While, the mobile phase for ergostane-type triterpenoids was a gradient eluting with a fixed ratio of $100 \%$ n-hexane $/ 100 \%$ ethylacetate $(55: 45)$ at a time interval of 0,20 and $30 \mathrm{~min}$.

\section{Preparation of triterpenoids standard solution and calibration curve}

Standard lanostane-type triterpenoids (dehydrosulphurenic acid and dehydroeburicoic acid) were kindly provided by Professor Yew-Min Tzeng of the Chaoyang University of Technology, Taichung, Taiwan, and ergostane-type triterpenoids (antcin A,B and C) were purified by HPLC partition from fruiting bodies of A. cinnamomea. Each standard $(2 \mathrm{mg})$ of dehydrosulphurenic acid and dehydroeburicoic acid was separately dissolved in $2 \mathrm{ml}$ of methanol while standards of antcin $\mathrm{A}$, antcin $\mathrm{B}$, and antcin $\mathrm{C}$ were dissolved in $2 \mathrm{ml}$ of ethyl acetate. Each dissolved solution (1.0 $\mathrm{mg}$ $\mathrm{ml} / 1$ ) was filtered through a $0.22 \mu \mathrm{m}$ filter (Millipore, USA) and further diluted as follows: 0.5 $\mathrm{mg} / \mathrm{ml}, 0.25 \mathrm{mg} / \mathrm{ml}, 0.125 \mathrm{mg} / \mathrm{ml}, 0.0625 \mathrm{mg} / \mathrm{ml}, 0.03125 \mathrm{mg} / \mathrm{ml}$. Standard solution $(20 \mu \mathrm{l})$ was used for HPLC injections. Calibration curves for standards were established, and high linearity (r2 $>0.999$ ) was obtained for each calibration curve. Calibration graphs were plotted based on linear regression analyses of the peak areas in response to concentrations of standards injected. The repeatability of the migration time and peak areas of dehydrosulphurenic acid, dehydroeburicoic acid, antcin $\mathrm{A}, \mathrm{B}$ and $\mathrm{C}$ were determined.

\section{Sample preparation for HPLC analysis}

Fruiting bodies (FB) and all mycelia samples subjected to HPLC analysis were prepared in two sets $(0.2 \mathrm{~g}$ each), freeze-dried for $24 \mathrm{~h}$ and powdered separately. One set in each group was dissolved in $30 \mathrm{ml}$ of methanol for lanostane-type triterpenoids, and another set was dissolved in 30 $\mathrm{ml}$ of ethanol for ergostane-type triterpenoids. These were ultrasonicated for $30 \mathrm{~min}$, and the supernatants were collected after centrifugation $(5000 \mathrm{rpm}, 10 \mathrm{~min})$. This process was repeated three times for each sample. After filtration, the combined extracts were evaporated to dryness with the help of a rotary evaporator. The residues of lanostane-type triterpenoids were dissolved in $5 \mathrm{ml}$ methanol separately. While, the residues of ergostane-type triterpenoids were dissolved in $5 \mathrm{ml}$ ethyl acetate separately. All the samples were filtered through a $0.22 \mu \mathrm{m}$ (Millipore, USA) 
membrane before analysis.

Table 1 HPLC analysis of fruiting bodies and mycelia of $A$. cinnamomea.

\begin{tabular}{llllll}
\hline \multirow{2}{*}{ Mycelium line } & $\begin{array}{l}\text { Lanostane-type } \\
\text { triterpenoids }\end{array}$ & & \multicolumn{3}{l}{$\begin{array}{l}\text { Ergostane-type } \\
\text { triterpenoids }\end{array}$} \\
\cline { 2 - 7 } & $\begin{array}{l}\text { Dehydrosulphurenic } \\
\text { acid (mg/g dw) }\end{array}$ & $\begin{array}{l}\text { Dehydroeburicoic } \\
\text { Acid (mg/g dw) }\end{array}$ & $\begin{array}{l}\text { Antcin-A } \\
\text { (mg/g dw) }\end{array}$ & $\begin{array}{l}\text { Antcin-B } \\
\text { (mg/g dw) }\end{array}$ & $\begin{array}{l}\text { Antcin-C } \\
\text { (mg/g dw) }\end{array}$ \\
\hline Fruiting bodies (WT) & $45.38 \pm 0.50$ & $7.40 \pm 0.12$ & $52.30 \pm 0.08$ & $49.90 \pm 0.35$ & $37.69 \pm 0.59$ \\
Mycelia (WT) & $3.89 \pm 0.30$ & $0.93 \pm 0.16$ & $0.38 \pm 0.06$ & $0.19 \pm 0.03$ & $*$ \\
GMM line H & $2.85 \pm 0.40$ & $2.06 \pm 0.38$ & $14.38 \pm 1.06$ & $13.12 \pm 1.27$ & $2.08 \pm 0.99$ \\
GMM line J-1 & $10.95 \pm 0.71$ & $4.27 \pm 0.42$ & $2.79 \pm 1.60$ & $3.65 \pm 0.84$ & $1.85 \pm 0.60$ \\
GMM line J-2 & $8.38 \pm 0.67$ & $2.15 \pm 0.37$ & $1.03 \pm 0.53$ & $2.18 \pm 0.67$ & $1.79 \pm 0.91$ \\
AD-1 & 23.22 & 7.44 & - & - & - \\
\hline
\end{tabular}

Mycelia cultured on MEA medium with $2 \%$ agar in $90 \mathrm{~mm}$ petridishes with agar blocks as supporting matrix. All data are means \pm S.D. of experiments performed in triplicate, except AD-1 related. * Could not be detected; - Not determined.

\section{Results and Discussion}

As evident from Fig.1 A-D, mycelia of control (WT-wild type) and three GMM (H, J-1, J-2) of A. cinnamomea showed different colors from off-white and light brown to dark brown. The amplification of the gus and hpt fragments with an expected size of 950 and $650 \mathrm{bp}$ respectively, in three GMM lines H, J-1 and J-2 (Fig.1.E) confirms their transgenic status. WT mycelia and nontemplate PCR reaction used as a negative control did not show any amplification. The mycelia of AD-1, the antisense mutant of DMATS (Dimethylallyl tryptophan synthase), showed pale white color and its gene-knock-out status was confirmed by PCR amplification (Fig.1.F).

HPLC analysis results of FB of WT A. cinnamomea, WT mycelia, three GMM (H, J-1, and J-2) and AD-1 are presented in Table 1. All mycelia were cultured on MEA medium with 2\% agar in $90 \mathrm{~mm}$ petridishes with agar blocks as supporting matrix. As expected, FB of WT $A$. cinnamomea showed much higher contents of all five triterpenoids analyzed (dehydrosulphurenic acid, dehydroeburicoic acid, antcin A, antcin B, and antcin C). In contrast to FB, the WT mycelia showed much lower amount of dehydrosulphurenic acid, dehydroeburicoic acid, almost undetectable antcin A, antcin B, and no antcin C. Surprisingly, all GMM when compared to WT mycelia not only contained higher dehydrosulphurenic acid (barring line $\mathrm{H}$ ) and dehydroeburicoic acid but also contained all three FB-specific triterpenoids (antcin A,B and C). Among them, there was a significantly higher amount of FB-specific triterpenoids in GMM line H. The highest dehydrosulphurenic acid content was recorded in GMM line J-1 (10.95 mg/g dw) and in control AD-1 (23.22 mg/g dw) compared to WT mycelia $(3.89 \mathrm{mg} / \mathrm{g} \mathrm{dw})$. The highest dehydroeburicoic acid content was also detected in GMM line J-1 (4.27 mg/g dw) and in control AD-1 (7.44 mg/g $\mathrm{dw})$ compared to WT mycelia $(0.93 \mathrm{mg} / \mathrm{g} \mathrm{dw})$. Among the three ergostane-type triterpenoids, the maximum content of antcin A (14.38 mg/g dw), antcin B (13.12 mg/g dw) and antcin C (2.08 mg/g $\mathrm{dw}$ ) were detected in the GMM line $\mathrm{H}$.

Although AD-1 showed higher lanostane-type triterpenoids in contrast to GMM, it was created as control and used to explain that by blocking DMATS (Dimethylallyl tryptophan synthase) in the terpenoid backbone biosynthesis pathway (upstream of triterpenoid biosynthesis pathway) (Fig.2), would enhance the accumulation of dimethylallyl diphosphate and improve flux to lanosterol (precursor of lanostane-type triterpenoids). Interestingly, the lack of DMATS in AD-1 made the mutant grow slowly (by measuring its mycelia diameter) and thinner (Fig.1 F). This made us unable to obtain sufficient sample material to perform triplicates and to analyze the content of its ergostane-type triterpenoids.

Medicinal fungi are becoming increasingly popular as functional foods in the last several years. It is estimated that between 80 to $85 \%$ of all medicinal fungi products are derived from fruiting bodies, which have been either artificially cultured or collected from the natural habitat (Lu et al. 2013). 

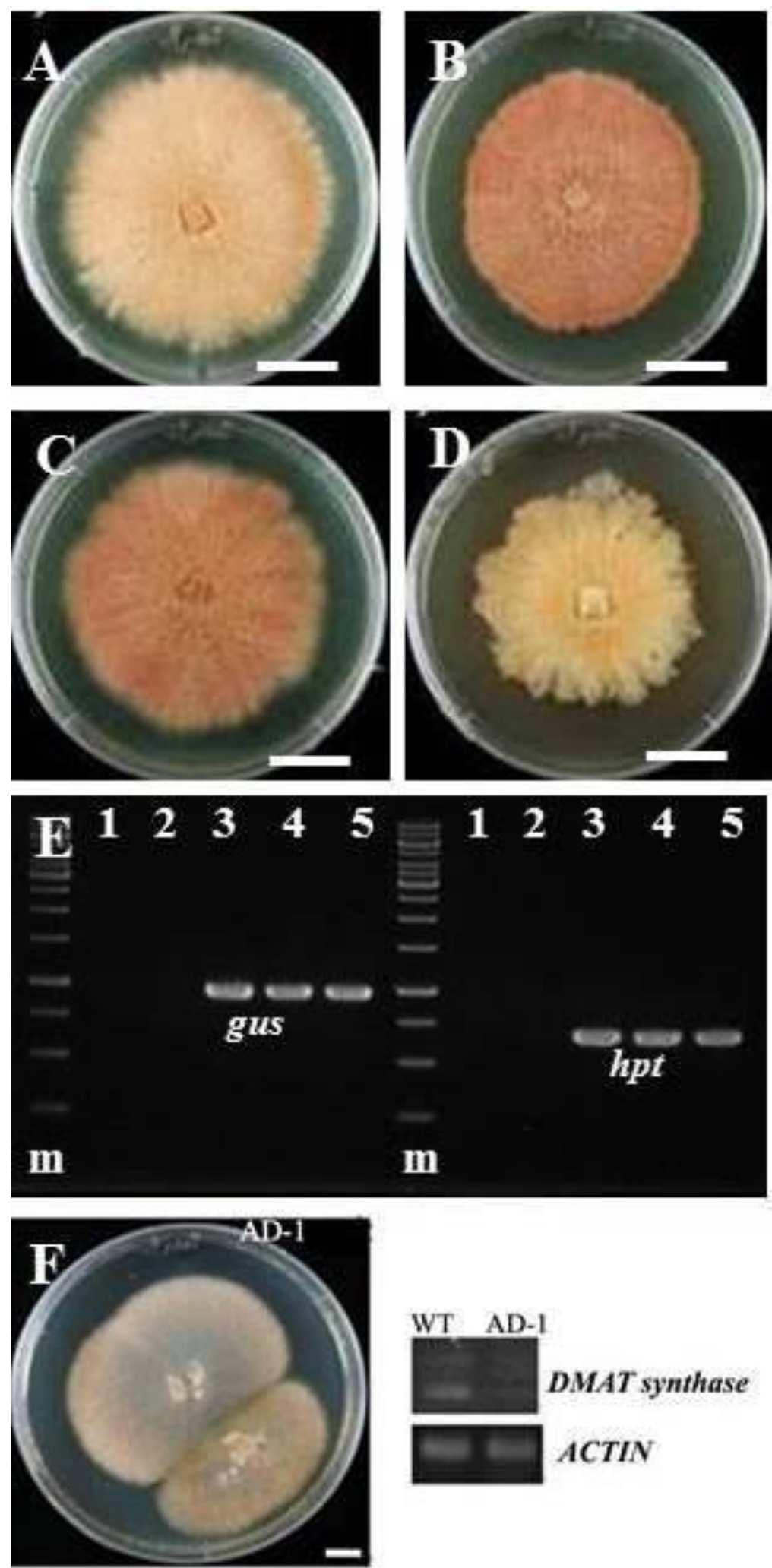

Fig. 1. A-F - Culture and PCR confirmation of WT mycelia and GMM of A. cinnamomea. A. WT strain BCRC 36401 of $A$. cinnamomea. B. GMM line H. C. GMM line J-1. D. GMM line J-2. E. PCR confirmation of integration of gus and hpt genes in the GMM lines: molecular marker (m), gus (950bp), hpt (650bp), lane1- Negative control, lane 2- WT, lane 3- GMM line H, lane 4- GMM line J-1, lane 5- GMM line J-2. F. Culture and PCR analysis of a gene-silenced mutant of $A$. cinnamomea strain BCRC 36401, AD-1 (35S::antisense dimethylallyl tryptophan synthase). Culture medium was MEA+2\% agar. All cultures were incubated in the dark. Bar $=2 \mathrm{~cm}(\mathrm{~A}-\mathrm{D}) ; 1$ $\mathrm{cm}(\mathrm{F})$. 
However, the extremely slow growth of A. cinnamomea, host specificity, rarity, use of only fruiting bodies (FB) and not mycelia as folk medicine, excessive collection from wild and ever growing demand are some of the factors which contribute to its limited supply and exorbitant cost. Therefore, it became imperative to develop alternative cultivation methods to meet the growing demand and to investigate if mycelia could produce medicinally important triterpenoids (mainly FB-specific). Cultivation of mycelia is much easier and faster compared to FB. Therefore, mycelial products have been considered as a great market potential in the future (Cheung 2008). In earlier reports, mycelia of $A$. cinnamomea could be cultivated in vitro, but these did not produce certain specific triterpenoids i.e. zhankuic acids $\mathrm{A}, \mathrm{B}, \mathrm{C}$, or antcin $\mathrm{K}$ which could be isolated only from FB of A. cinnamomea, and considered to be effective compounds for curing certain illnesses (Shen et al. 2004). Therefore, one of the options could be the induction of bioactive compounds in the mycelia of $A$. cinnamomea by activation tagging method. Out of several GMM lines created, we selected GMM line H, J-1 and J-2 showing higher growth rate and carried out HPLC analysis. All these GMM lines showed FB-specific triterpenoids.

In contrast to WT mycelia (Fig.1A), the culture of GMM line $\mathrm{H}$ and $\mathrm{J}-1$ showed a similar growth pattern, texture, and color (Fig. 1B, C). However, there was a significant difference in contents of the type of triterpenoids in them, and it has no bearing on the mycelia color. Among all three GMM lines, $\mathrm{H}$ contained the highest levels of ergostane type triterpenoids. The amount of antcin A (14.38 mg/g dw) and B (13.12 mg/g dw) detected in its mycelia almost reached $1 / 4$ of those in the FB of WT A. cinnamomea. Although GMM line J-1, and another GMM line J-2 (Fig. 1D) with relatively slower growth also produced FB-specific triterpenoids in mycelia, but their contents were significantly higher compared to WT mycelia. These results indicate that every GMM line is different with respect to growth and compound profiles. The unique genetic modified background (caused by random activation-tagging) of each GMM would possibly change any flux of the triterpenoid biosynthetic pathway, as shown in the control AD-1. And any change in such pathway would interfere mycelia growth rate. Several mycologists (Lee et al. 2002, Sunagawa \& Magae 2005, Yamada et al. 2006) have made efforts to study gene expression during the formation of fruit bodies, porous-hymenium basidiomatal. However, formation and biosynthesis of active compounds at the molecular level have not yet been explained. Theoretically, the gene(s) altered in activation tagged GMM lines $\mathrm{H}, \mathrm{J}-1$ and $\mathrm{J}-2$ could possibly related to terpenoid backbone biosynthesis pathway (Fig. 2), so we had created a 35S::antisense mutant AD-1, used as a control in the present study. However, the morphology of AD-1 mycelia did not look like any of $\mathrm{H}, \mathrm{J}-1$ and J-2, and the tritepenoid contents between them were also different. Based on these results, more mutants should be created in the further studies in order to discover unknown biosynthesis pathway of lanostane and ergostane-type triterpenoids in A. cinnamomea. Lepesheva \& Waterman (2007) had demonstrated that lanosterol $14 \alpha$-demethylase (CYP51) is a necessary enzyme for sterol biosyntheses, so an overexpressed mutant of CYP51 in A. cinnamomea would be created for further studies (Chu et al. 2008, Chu \& Chang 2007).

Totally three GMM lines with FB-specific triterpenoids were identified in this study. Though the contents of all three antcins (A, B, and C) in GMM line H, J-1 and J-2 were lower than the fruiting bodies of WT A. cinnamomea, however, present findings have significance in that, by an application of activation tagging it is feasible to induce and/or enhance the production of FBspecific triterpenoids in mycelia of this medicinally important fungus.

The present study demonstrates that through genetic engineering (or gene modification), it is possible to induce/enhance FB-specific triterpenoids (antcin-A, B and C), and lanostane-type triterpenoids (dehydroeburicoic acid and dehydrosulphurenic acid) which are normally found abundantly in FB of A. cinnamomea. For further studies, a large-scale production of these GMM should be performed in order to monitor any culture condition that could affect the quantities of the FB-specific triterpenoids. Our findings in this study will help the production of mycelia which are becoming increasingly popular as an important functional food for the treatment of several critical illnesses including cancer. 


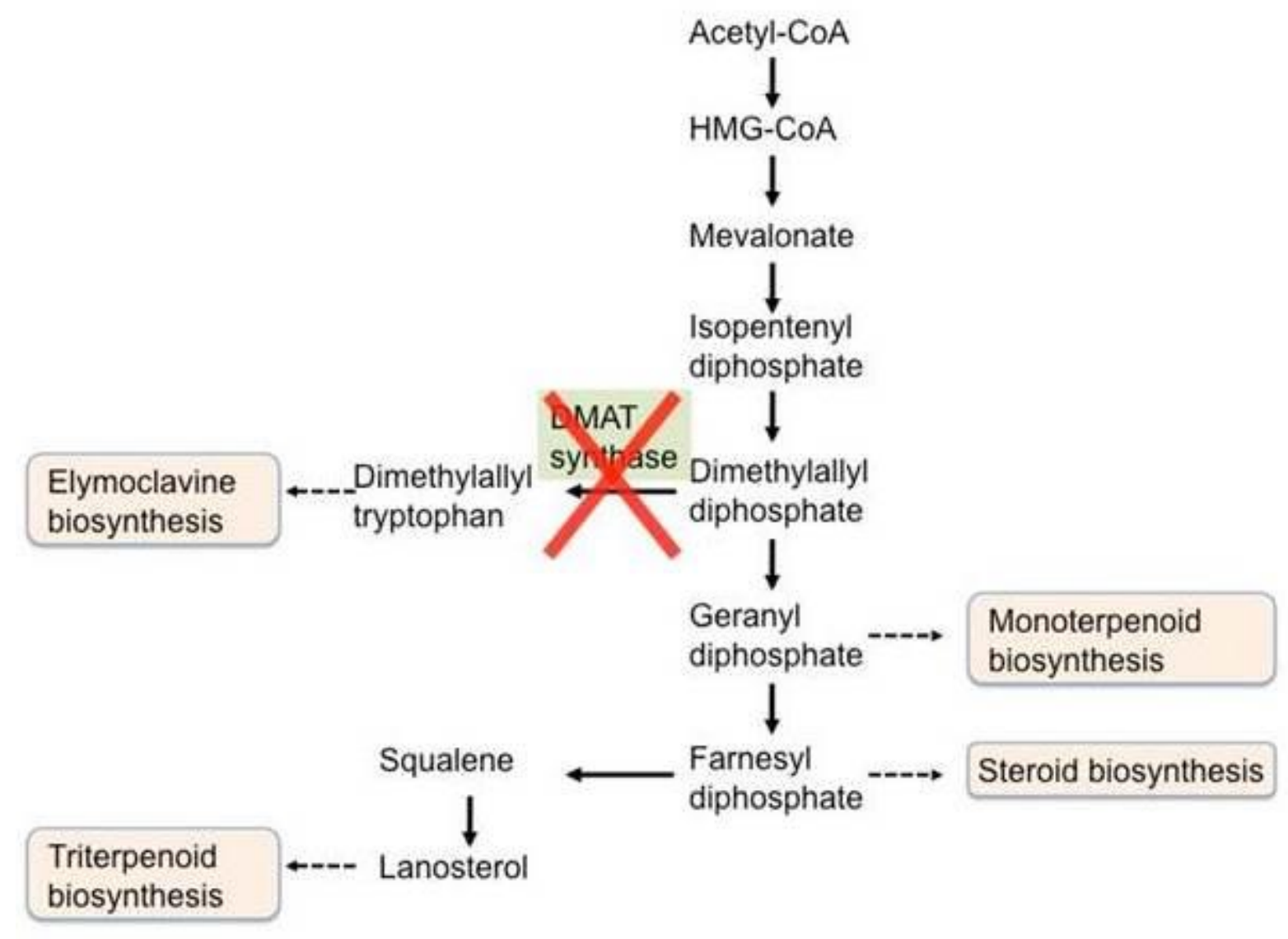

Fig. 2 - Illustration of the blocked terpenoid backbone biosynthesis pathway in $35 \mathrm{~S}$ antisense DMAT synthase mutant, AD-1. Illustration according to the information provided by KEGG (http://www.kegg.jp/kegg-bin/show_pathway?map00900).

\section{Competing interests}

We declare that there are no competing interests among the authors and the funding agency.

\section{Acknowledgements}

We thank Professor Yew-Min Tzeng of the Chaoyang University of Technology, Taichung, Taiwan for providing the standard triterpenoid compounds, Professor ShengYang Wang of National Chung Hsing University for providing the fruiting bodies of A. cinnamomea and Professor $\mathrm{Su}-\mathrm{Mei} \mathrm{Yu}$, Academica Sinica, Taipei for providing the activation tagging plasmid pTAG-8 for the present study. Financial assistance for the research program (98 Agriscience-1.2.1-Science-aj) provided by Council of Agriculture, Taiwan is gratefully acknowledged.

\section{References}

Busov VB, Meilan R, Pearce DW, Ma C, Rood SB, Strauss SH. 2003 - Activation tagging of a dominant gibberellin catabolism gene (GA 2-oxidase) from popular that regulates tree stature. Plant Physiology 132, 1283-1291. doi: http://dx.doi.org/10.1104/pp.103.020354.

Chang TT, Chou WN. 2004 - Antrodia cinnamomea reconsidered and A. salmonea sp. nov. on Cunninghamia konishii in Taiwan. Botanical Bulletin of Academia Sinica 45, 347-352.

Chen C, Liu YW, Ker YB, Wu YY, Lai EY, Chyau CC, Hseu TH, Peng RY. 2007 - Chemical characterization and anti-inflammatory effect of polysaccharides fractionated from submerge-cultured Antrodia camphorata mycelia. Journal of Agricultural and Food Chemistry 55,5007-5012. doi:10.1021/jf063484c. 
Chen CC, Shiao YJ, Lin RD, Shao YY, Lai MN, Lin CC, Ng LT, Kuo YH. 2006 -Neuroprotective diterpenes from the fruiting body of Antrodia camphorata. Journal of Natural Products 69, 689-691. doi: 10.1021/np0581263.

Chen ECF, Su YH, Kanagarajan S, Agrawal DC, Tsay HS. 2009 - Development of activation tagging system for the basidiomycetous medicinal fungus Antrodia cinnamomea. Mycological Research 113, 290-297. doi:10.1016/j.mycres.2008.11.007.

Cheung PCK. 2008 - Overview of mushroom cultivation and utilization as functional foods. In: Cheung PCK (ed) Mushrooms as Functional Foods. John Wiley \& Sons, Hoboken, NJ

Chiang CC, Chiang BH. 2013 - Processing characteristics of submerged fermentation of Antrodia cinnamomea in airlift bioreactor. Biochemical Engineering Journal 73, 65-71. http://dx.doi.org/10.1016/j.bej.2013.01.016.

Chu FH, Chang TT. 2007 - Basidiomatal formation in Antrodia cinnamomea from the perspective of gene expression. Botanical Studies 48,387-396.

Chu FH, Lee YR, Chou SJ, Chang TT, Shaw JF. 2008 - Isolation and analysis of genes specifically expressed during basidiomatal development in Antrodia cinnamomea by subtractive PCR and cDNA microarray. FEMS Microbiology Letters 280,150-159. doi: 10.1111/j.15746968.2008.01052.x.

Fa KN, Yang CM, Chen PC, Lee YY, Chyau CC, Hu ML. 2015 - Anti-metastatic effects of antrodan, the Antrodia cinnamomea mycelia glycoprotein, in lung carcinoma cells. International Journal of Biological Macromolecules 74, 476-482. http://dx.doi.org/10.1016/j.ijbiomac.2015.01.004.

Geethangili M, Tzeng YM. 2011 - Review of pharmacological effects of Antrodia camphorata and its bioactive compounds. Evidence-Based Complementary \& Alternative Medicine 2011, 117. http://dx.doi.org/10.1093/ecam/nep108.

Hseu YC, Chen SC, Tsai PC, Chen CS, Lu FJ, Chang NW, Yang HL 2007 - Inhibition of cyclooxygenase-2 and induction of apoptosis in estrogen-nonresponsive breast cancer cells by Antrodia camphorata. Food and Chemical Toxicology 45, 1107-1115. doi:10.1016/j.fct.2006.12.012.

Hsiao G, Shen MY, Lin KH, Lan MH, Wu LY, Chou DS, Lin CH, Su CH, Sheu JR. 2003 Antioxidative and hepatoprotective effects of Antrodia camphorata extract. Journal of Agricultural and Food Chemistry 51, 3302-3308. doi: 10.1021/jf021159t.

Hsing YI, Chern CG, Fan MJ, Lu PC, Chen KT, et al. 2007 - A rice gene activation/knockout mutant resource for high throughput functional genomics. Plant Molecular Biology 63, 351-364. doi: 10.1007/s11103-006-9093-z.

Hsu YL, Kuo PL, Cho CY, Ni WC, Tzeng TF, Ng LT, Kuo YH, Lin CC. 2007 - Antrodia cinnamomea fruiting bodies extract suppresses the invasive potential of human liver cancer cell line PLC/PRF/5 through inhibition of nuclear factor kappaB pathway. Food and Chemical Toxicology 45, 1249-1257. doi:10.1016/j.fct.2007.01.005.

Hsu YL, Kuo YC, Kuo PL, Ng LT, Kuo YH, Lin CC. 2005 - Apoptotic effects of extract from Antrodia camphorata fruiting bodies in human hepatocellular carcinoma cell lines. Cancer Letters 221, 77-89. doi:10.1016/j.canlet.2004.08.012.

Huang SJ, Mau JL. 2007 - Antioxidant properties of methanolic extracts from Antrodia camphorata with various doses of $\gamma$-irradiation. Food Chemistry 105, 1702-1710. doi:10.1016/j.foodchem.2007.04.046.

Kardailsky I, Shukla VK, Ahn JH, Dagenais N, Christensen SK, Nguyen JT, Chory J, Harrison MJ, Weigel D. 1999 - Activation tagging of the floral inducer FT. Science 286, 1962-1965. doi: 10.1126/science.286.5446.1962.

Kuo PL, Hsu YL, Cho CY, Ng LT, Kuo YH, Lin CC. 2006 - Apoptotic effects of Antrodia cinnamomea fruiting bodies extract are mediated through calcium and calpain dependent pathways in Hep 3B cells. Food and Chemical Toxicology 44, 1316-1326. doi:10.1016/j.fct.2006.02.009. 
Lee SH, Kim BG, Kim KJ, Lee JS, Yun DW, Hahn JH, Kim GH, Lee KH, Suh DS, Kwon ST, Lee CS, Yoo YB. 2002 - Comparative analysis of sequences expressed during the lipid-cultured mycelia and fruit body stages of Pleurotus ostreatus. Fungal Genetics and Biology 35, 115134. doi:10.1006/fgbi.2001.1310.

Liu JJ, Huang TS, Hsu ML, Chen CC, Lin WS, Lu FJ, Chang WH. 2004 - Antitumor effects of the partially purified polysaccharides from Antrodia camphorata and the mechanism of its action. Toxicology and Applied Pharmacology 201, 186-193. doi:10.1016/j.taap.2004.05.016.

Liu Z, Liang HJ, Chen CH, Su CH, Lee TH, Huang CT, Hou WC, Lin SY, Zhong WB. 2007 Comparative anti-inflammatory characterization of wild fruiting body, liquid-state fermentation, and solid-state culture of Taiwanofungus camphoratus in microglia and the mechanism of its action. Journal of Ethnopharmacology 113, 45-53. doi:10.1016/j.jep.2007.03.037.

Lu MC, Mohamed ES, Wu TY, Du YC, Chang TT, Chen CF, Hsu YM, Lai KH, Chiu CP, Chang FR, Wu YC. 2013 - Recent research and development of Antrodia cinnamomea. Pharmacology \& Therapeutics 139, 124-156.

Mathews H, Clendennen SK, Caldwell CG, Liu XL, Connors K, Matheis N, Schuster DK, Menasco DJ, Wagoner W, Lightner J, Wagner DR. 2003 - Activation tagging in tomato identifies a transcriptional regulator of anthocyanin biosynthesis, modification, and transport. Plant Cell 15, 1689-1703. doi: http://dx.doi.org/10.1105/tpc.012963.

Mau JL, Huang PN, Huang SJ, Chen CC. 2004 - Antioxidant properties of methanolic extracts from two kinds of Antrodia camphorata mycelia. Food Chemistry 86, 25-31. doi:10.1016/j.foodchem.2003.08.025.

Nakamura N, Hirakawa A, Gao JJ, Kakuda H, Shiro M, Komatsu Y, Sheu CC, Hattori M. 2004 Five new maleic and succinic acid derivatives from the mycelium of Antrodia camphorata and their cytotoxic effects on LLC tumor cell line. Journal of Natural Products 67, 46-48. doi: 10.1021/np030293k.

Shen YC, Wang YH, Chou YC, Chen CF, Lin LC, Chang TT, Tien JH, Chou CJ. 2004 Evaluation of the anti-inflammatory activity of zhankuic acids isolated from the fruiting bodies of Antrodia camphorata. Planta Medica 70, 310-314. doi: 10.1055/s-2004-818941.

Song TY, Hsu SL, Yen GC. 2005 - Induction of apoptosis in human hepatoma cells by mycelia of Antrodia camphorata in submerged culture. Journal of Ethnopharmacology 100, 158-167. doi:10.1016/j.jep.2005.02.043.

Song TY, Yen GC. 2002 - Antioxidant properties of Antrodia camphorata in submerged culture. Journal of Agricultural and Food Chemistry 50, 3322-3327. doi: 10.1021/jf011671z.

Song TY, Yen GC. 2003 - Protective effects of fermented filtrate from Antrodia camphorata in submerged culture against CCl4-induced hepatic toxicity in rats. Journal of Agricultural and Food Chemistry 51, 1571-1577. doi: 10.1021/jf904257h.

Sunagawa M, Magae Y. 2005 - Isolation of genes differentially expressed during the fruit body development of Pleurotus ostreatus by differential display of RAPD. FEMS Microbiology Letters 246, 279-284. doi: http://dx.doi.org/10.1016/j.femsle.2005.04.018.

Tsai TC, Tung YT, Kuo YH, Liao JW, Tsai HC, Chong KY, Chen HL, Chen CM. 2015 - Antiinflammatory effects of Antrodia camphorata, a herbal medicine, in a mouse skin ischemia model. Journal of 113-121. http://dx.doi.org/10.1016/j.jep.2014.11.015.

Tsai ZT, Liaw SL. 1982 - The use and the Effect of Ganoderma. Sheng-Tun Publisher: Taichung, Taiwan, pp. 116-117.

Van der Fits L, Hilliou F, Memelink J. 2001 - T-DNA activation tagging as a tool to isolate regulators of a metabolic pathway from a genetically non-tractable plant species. Transgenic Research 10, 513-521. doi:10.1023/A:1013087011562. 
Wang J, Tseng HW, Chou CJ, Tsai TH, Chen CT, Lu MK. 2003 - The vasorelaxation of Antrodia camphorata mycelia: involvement of endothelial $\mathrm{Ca}^{2+}-\mathrm{NO}-\mathrm{cGMP}$ pathway. Life Science 73, 2769-2783. doi:10.1016/S0024-3205(03)00669-6.

Yamada M, Sakuraba S, Shibata K, Taguchi G, Inatomi S, Okazaki M, Shimosaka M. 2006 Isolation and analysis of genes specifically expressed during fruiting body development in the basidiomycete Flammulina velutipes by fluorescence differential display. FEMS Microbiology Letters 254, 165-172. doi: http://dx.doi.org/10.1111/j.15746968.2005.00023.x.

Yang L, Chen CS, Chang WH, Lu FJ, Lai YC, Chen CC, Hseu TH, Kuo CT, Hseu YC. 2006 Growth inhibition and induction of apoptosis in MCF-7 breast cancer cells by Antrodia camphorata. Cancer Letters 231, 215-227. doi:10.1016/j.canlet.2005.02.004. 